\title{
Assessment of Haar Wavelet-Quasilinearization Technique in Heat Convection-Radiation Equations
}

\author{
Umer Saeed and Mujeeb ur Rehman \\ School of Natural Sciences, National University of Sciences and Technology, Sector H-12, Islamabad, Pakistan \\ Correspondence should be addressed to Umer Saeed; umer.math@gmail.com
}

Received 31 August 2013; Revised 25 November 2013; Accepted 26 December 2013; Published 5 February 2014

Academic Editor: Francesco Morabito

Copyright (C) 2014 U. Saeed and M. ur Rehman. This is an open access article distributed under the Creative Commons Attribution License, which permits unrestricted use, distribution, and reproduction in any medium, provided the original work is properly cited.

\begin{abstract}
We showed that solutions by the Haar wavelet-quasilinearization technique for the two problems, namely, (i) temperature distribution equation in lumped system of combined convection-radiation in a slab made of materials with variable thermal conductivity and (ii) cooling of a lumped system by combined convection and radiation are strongly reliable and also more accurate than the other numerical methods and are in good agreement with exact solution. According to the Haar wavelet-quasilinearization technique, we convert the nonlinear heat transfer equation to linear discretized equation with the help of quasilinearization technique and apply the Haar wavelet method at each iteration of quasilinearization technique to get the solution. The main aim of present work is to show the reliability of the Haar wavelet-quasilinearization technique for heat transfer equations.
\end{abstract}

\section{Introduction}

Haar wavelet is the lowest member of Daubechies family of wavelets and is convenient for computer implementations due to availability of explicit expression for the Haar scaling and wavelet functions [1]. The quasilinearization approach was introduced by Bellman and Kalaba [2] as a generalization of the Newton-Raphson method to solve the individual or systems of nonlinear ordinary and partial differential equations.

Haar wavelet-quasilinearization technique [3-6] is recently developed method for the nonlinear differential equation, which deals with all types of nonlinearities. Boundary value problems are considerably more difficult to deal with than initial value problems. The Haar wavelet method for boundary value problems is more complicated than for initial value problems. In the present work we deal with both initial and boundary value problems.

In this present work, our purpose to solve the nonlinear equations arising in heat transfer through Haar waveletquasilinearization technique and show that it is strongly reliable method for heat transfer problems than the other existing methods. Convergence of Haar wavelet-quasilinearization technique has been given in [6].
We use the cubic spline interpolation [7] to get the solution at grid points for the sake of comparison. For this purpose we use the MATLAB built-in function $y_{i}=$ interp1 $\left(x, y, x_{i}\right.$, "spline"), for one-dimensional data interpolation by cubic spline interpolation.

The paper is arranged as follows: in Section 2 we review basic definition of fractional differentiation and integration, while in Section 3 we describe the Haar wavelets. In Section 4 we present the main features of the quasilinearization approach. In Section 5 we apply the Haar wavelet method with quasilinearization technique to nonlinear heat transfer problems. Finally in Section 6 we conclude our work.

\section{Preliminaries}

In this section, we review basic definitions of fractional differentiation and fractional integration [8].

2.1. Riemann-Liouville Fractional Integral Operator of Order $\alpha$. The operator $I_{x}^{\alpha}$, defined on $L_{1}[a, b]$ by

$$
I_{x}^{\alpha} y(x)=\frac{1}{\Gamma(\alpha)} \int_{0}^{x}(x-t)^{\alpha-1} y(t) d t
$$


for $a \leq x \leq b$, where $\alpha \in \mathbb{R}^{+}$, is called Riemann-Liouville fractional integral of order $\alpha$.

2.2. Riemann-Liouville and Caputo Fractional Derivative Operator of Order $\alpha$. The operator $D_{x}^{\alpha}$, defined by

$$
D_{x}^{\alpha} y(x)=\frac{1}{\Gamma(n-\alpha)}\left(\frac{d}{d x}\right)^{n} \int_{0}^{x}(x-t)^{n-\alpha-1} y(t) d t
$$

for $a \leq x \leq b$, where $\alpha \in \mathbb{R}^{+}$and $n=\lceil\alpha\rceil$, is called RiemannLiouville fractional derivative of order $\alpha$.

The Caputo fractional derivative of a function $y \in$ $L_{1}[a, b]$ is defined as

$$
{ }^{c} D_{x}^{\alpha} y(x)=\frac{1}{\Gamma(n-\alpha)} \int_{0}^{x}(x-t)^{n-\alpha-1}\left(\frac{d}{d t}\right)^{n} y(t) d t,
$$

for $a \leq x \leq b$, where $\alpha \in \mathbb{R}^{+}$and $n=\lceil\alpha\rceil$.

\section{The Haar Wavelets}

The Haar function contains just one wavelet during some subinterval of time and remains zero elsewhere and is orthogonal. The uniform Haar wavelets are useful for the treatment of solution of differential equations which have no abrupt behavior. The $i$ th uniform Haar wavelet $h_{i}(x), x \in$ $[a, b]$, is defined as follows [9]:

$$
h_{i}(x)= \begin{cases}1, & \frac{k}{m} \leq x<\frac{k+0.5}{m} \\ -1, & \frac{k+0.5}{m} \leq x<\frac{k+1}{m} \\ 0, & \text { otherwise }\end{cases}
$$

where $i=2^{j}+k+1, j=0,1,2, \ldots, J$ is dilation parameter, $m=2^{j}$, and $k=0,1,2, \ldots, 2^{j}-1$ is translation parameter. $J$ is maximal level of resolution and the maximal value of $i$ is $2 M$ where $M=2^{J}$. In particular, $h_{1}(x):=\chi_{[a, b]}(x)$, where $\chi_{[a, b]}(x)$ is characteristic function on interval $[a, b]$, is the Haar scaling function. For the uniform Haar wavelet, the wavelet-collocation method is applied. The collocation points for the Haar wavelets are usually taken as $x_{j}=(j+0.5) / 2 M$, where $j=1,2, \ldots, 2 M$.

3.1. Integral of the Haar Wavelets. Any function $y \in L_{2}[a, b]$ can be represented in terms of the Haar series:

$$
\begin{aligned}
& y(x)=\sum_{l=1}^{\infty} b_{l} h_{l}(x), \quad l=2^{j}+k+1, \\
& j=0,1,2, \ldots, J, \quad k=0,1,2, \ldots, 2^{j}-1,
\end{aligned}
$$

where $b_{l}$ are the Haar wavelet coefficients given as $b_{l}=$ $\int_{-\infty}^{\infty} y(x) h_{l}(x) d x$.
The Riemann-Liouville fractional integral of the Haar wavelets is given as

$$
I_{x}^{\alpha} h_{1}(x)=\frac{(x-a)^{\alpha}}{\Gamma(\alpha+1)}
$$

$$
\begin{aligned}
& P_{\alpha, l}(x) \\
& =I_{x}^{\alpha} h_{l}(x) \\
& =\frac{1}{\Gamma(\alpha)} \int_{a}^{x}(x-s)^{\alpha-1} h_{l}(s) d s \\
& =\frac{1}{\Gamma(\alpha+1)}\left\{\begin{array}{cc}
(x-a(l))^{\alpha}, & a(l) \leq x<b(l) ; \\
(x-a(l))^{\alpha}-2(x-b(l))^{\alpha}, 2(x-b(l))^{\alpha}, & b(l) \leq x<c(l) ; \\
+(x-c(l))^{\alpha}, & x \geq c(l) .
\end{array}\right.
\end{aligned}
$$

\section{Quasilinearization [2]}

The quasilinearization approach is a generalized NewtonRaphson technique for functional equations. It converges quadratically to the exact solution, if there is convergence at all, and it has monotonic convergence.

Let us consider the nonlinear $n$th order differential equation

$$
L^{n} y(x)=f\left(y(x), y^{\prime}(x), \ldots, y^{n-1}(x), x\right)
$$

Application of quasilinearization technique to (7) yields

$$
\begin{aligned}
L^{n} y_{r+1}(x) & \\
= & f\left(y_{r}(x), y_{r}^{\prime}(x), \ldots, y_{r}^{n-1}(x), x\right) \\
& +\sum_{j=0}^{n-1}\left(y_{r+1}^{j}(x)-y_{r}^{j}(x)\right) f_{y^{j}}\left(y_{r}(x), y_{r}^{\prime}(x), \ldots,\right. \\
& \left.y_{r}^{n-1}(x), x\right),
\end{aligned}
$$

with the initial/boundary conditions at $(r+1)$ th iteration, where $n$ is the order of the differential equation. Equation (8) is always a linear differential equation and can be solved recursively, where $y_{r}(x)$ is known and one can use it to get $y_{r+1}(x)$.

\section{Applications}

5.1. Temperature Distribution Equation in Lumped System of Combined Convection-Radiation in a Slab Made of Materials with Variable Thermal Conductivity. Let the lumped system have volume $V$, surface area $A$, density $\rho$, specific heat $c$, initial temperature $T_{i}$, temperature of the convection environment $T_{a}$, heat transfer coefficient $h$, and $c_{a}$ which is specific heat at temperature $T_{a}$. Consider that the mathematical model describing the temperature distribution in lumped system of combined convection-radiation in a slab made of 
TABLE 1: Numerical results for temperature distribution equation for $\varepsilon=0.6$ : Haar wavelet-quasilinearization technique at 4 th iteration and level of resolutions $J=8$.

\begin{tabular}{|c|c|c|c|c|}
\hline \multicolumn{5}{|c|}{4 th iteration $(J=8)$} \\
\hline$x$ & Maple & $y_{\mathrm{GA}}[10]$ & $y_{\text {HPM }}[10]$ & $y_{\text {Haar }}$ \\
\hline 0.0 & 0.834542 & 0.963536 & 0.640000 & 0.834543 \\
\hline 0.2 & 0.840390 & 0.964009 & 0.652096 & 0.840391 \\
\hline 0.4 & 0.858269 & 0.965742 & 0.689536 & 0.858269 \\
\hline 0.6 & 0.889247 & 0.969893 & 0.755776 & 0.889248 \\
\hline 0.8 & 0.935346 & 0.979233 & 0.866576 & 0.935346 \\
\hline
\end{tabular}

materials with variable thermal conductivity is given by the following nonlinear boundary value problem:

$$
\begin{gathered}
\frac{d^{2} y(x)}{d x^{2}}-\varepsilon y^{4}(x)=0, \quad 0 \leq x \leq 1, \\
\frac{d y(0)}{d x}=0, \quad y(1)=1,
\end{gathered}
$$

where $y=\left(T-T_{a}\right) /\left(T_{i}-T_{a}\right)$ is dimensionless temperature, $x=t /\left(\rho V c_{a} / h A\right)$ is dimensionless time, and $\varepsilon=\beta\left(T-T_{a}\right)$.

5.1.1. Haar Wavelet-Quasilinearization Technique. Applying the quasilinearization technique to (9), we get

$$
\begin{gathered}
\frac{d_{n+1}^{2} y(x)}{d x^{2}}-4 \varepsilon y_{n}^{3}(x) y_{n+1}(x)=-3 \varepsilon y_{n}^{4}(x), \quad 0 \leq x \leq 1, \\
\frac{d y_{n+1}(0)}{d x}=0, \quad y_{n+1}(1)=1 .
\end{gathered}
$$

Now we implement the Haar wavelet method to (10); we approximate the higher-order derivative term by the Haar wavelet series as

$$
\frac{d^{2} y_{n+1}(x)}{d x^{2}}=\sum_{l=1}^{2 M} b_{l} h_{l}(x) .
$$

Lower-order derivatives are obtained by integrating (11) and using the boundary conditions:

$$
y_{n+1}(x)=\sum_{l=1}^{2 M} b_{l} p_{2, l}(x)-C_{2, l}+1,
$$

where $C_{2, l}=\int_{0}^{1} p_{2, l}(x) d x$.

Substitute (11) and (12) in (10) to obtain

$$
\begin{gathered}
\sum_{l=1}^{2 M} b_{l}\left[h_{l}(x)-4 \varepsilon y_{n}^{3}(x) p_{2, l}(x)+4 \varepsilon y_{n}^{3}(x) C_{2, l}\right] \\
=-3 \varepsilon y_{n}^{4}(x)+4 \varepsilon y_{n}^{3}(x),
\end{gathered}
$$

with the initial approximation $y_{0}(x)=0$.

Figure 1 shows the temperature $y_{\text {Haar }}$ by Haar waveletquasilinearization technique for different $\varepsilon$ at $J=5$ and at 4 th

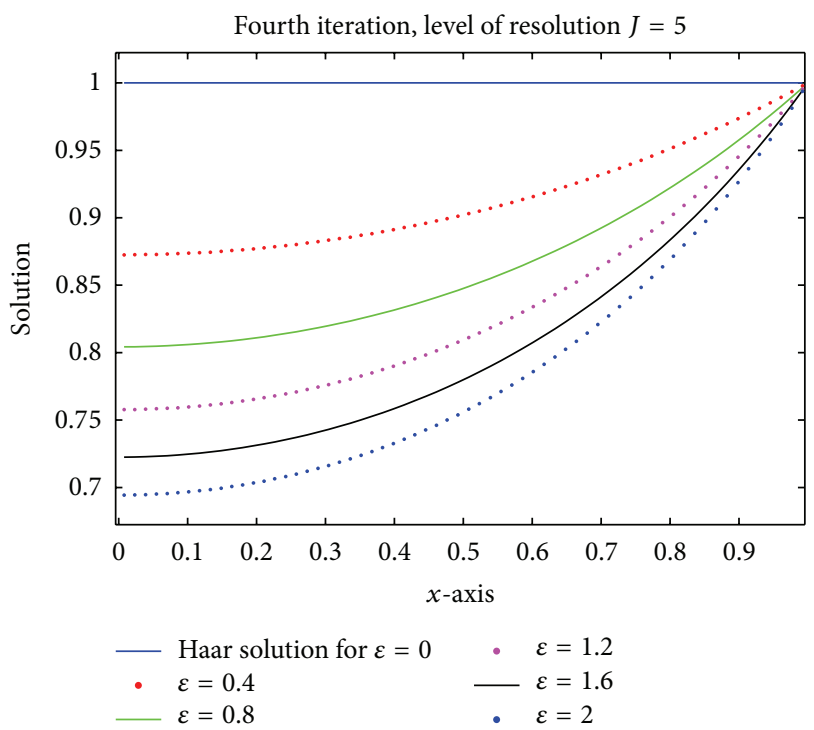

Figure 1: Solutions by Haar wavelet-quasilinearization technique for different $\varepsilon$ at $J=5$ and $n=4$.

iteration. According to Figure 1 and Tables 1 and 2, temperature increases with decreasing $\varepsilon$; also temperature varies with time $x$. Tables 1 and 2 show that the obtained solutions are in good agreement with the numerical solution provided by Maple and are better than generalized approximation method $y_{\mathrm{GA}}[10]$ and homotopy perturbation method $y_{\mathrm{HPM}}[10]$.

5.2. Cooling of a Lumped System by Combined Convection and Radiation. Consider that the system has volume $V$, surface area $A$, density $\rho$, specific heat $c$, emissivity $E$, initial temperature $T_{i}$, temperature of the convection environment $T_{a}$, heat transfer coefficient $h$, and $c_{a}$ which is specific heat at temperature $T_{a}$. In this case system loses heat through radiation and the effective sink temperature is $T_{s}$. The mathematical model describing the cooling of a lumped system by combined convection and radiation is given by the following nonlinear initial value problem:

$$
\begin{array}{r}
\rho V c \frac{d T(t)}{d t}+h A\left(T-T_{a}\right)+E \sigma A\left(T^{4}-T_{s}^{4}\right)=0, \\
T(t=0)=T_{i} .
\end{array}
$$


TABLE 2: Numerical results for temperature distribution equation for $\varepsilon=2.0$ : Haar wavelet-quasilinearization technique at 4 th iteration and level of resolutions $J=8$.

\begin{tabular}{lcccc}
\hline & & 4 th iteration $(J=8)$ & $y_{\text {HPM }}[10]$ & $y_{\text {Haar }}$ \\
$x$ & Maple & $y_{\mathrm{GA}}[10]$ & -0.666667 & 0.694362 \\
0.0 & 0.694318 & 0.968771 & -0.625600 & 0.703739 \\
0.2 & 0.703698 & 0.968804 & -0.489600 & 0.732927 \\
0.4 & 0.732894 & 0.969008 & -0.220267 & 0.785510 \\
0.6 & 0.785488 & 0.970024 & -0.246400 & 0.869176 \\
0.8 & 0.869161 & 0.975059 & & \\
\hline
\end{tabular}

For the solution of (14), we do the following certain changes in parameters:

$$
\begin{gathered}
y=\frac{T}{T_{i}}, \quad y_{a}=\frac{T_{a}}{T_{i}}, \quad y_{s}=\frac{T_{s}}{T_{i}}, \\
x=\frac{t}{\rho V c_{a} / h A}, \quad \varepsilon=\frac{E \sigma T_{i}^{3}}{h} .
\end{gathered}
$$

Equation (14) implies after changing the parameters

$$
\begin{array}{r}
\frac{d y(x)}{d x}+\left(y-y_{a}\right)+\varepsilon\left(y^{4}-y_{s}^{4}\right)=0, \\
y(x=0)=1 .
\end{array}
$$

For the sake of simplicity we assume that $y_{a}=y_{s}=0$, (15) becomes

$$
\begin{array}{r}
\frac{d y(x)}{d x}+y+\varepsilon y^{4}=0, \\
y(x=0)=1 .
\end{array}
$$

5.2.1. Haar Wavelet-Quasilinearization Technique. Implementation of the quasilinearization technique to (16) gives

$$
\begin{array}{r}
\frac{d y_{n+1}(x)}{d x}+\left(1+4 \varepsilon y_{n}^{3}\right) y_{n+1}=3 \varepsilon y_{n}^{4}, \\
y_{n+1}(x=0)=1 .
\end{array}
$$

According to the Haar wavelet method to (18), approximate the higher-order derivative term by the Haar wavelet series as

$$
\frac{d y(x)}{d x}=\sum_{l=1}^{2 M} b_{l} h_{l}(x) .
$$

Solution can be obtained by integrating (19) and using the initial condition to yield

$$
y_{n+1}(x)=\sum_{l=1}^{2 M} b_{l} p_{1, l}(x)+1 .
$$

Substituting (19) and (20) in (18),

$$
\begin{gathered}
\sum_{l=1}^{2 M} b_{l}\left[h_{l}(x)+\left(1+4 \varepsilon y_{n}^{3}(x)\right) p_{1, l}(x)\right] \\
=3 \varepsilon y_{n}^{4}(x)-\left(1+4 \varepsilon y_{n}^{3}(x)\right),
\end{gathered}
$$

with the initial approximation $y_{0}(x)=1$.
To get the solution on large interval, say $[0,5]$, we divide the interval $[0,5]$ into three subintervals $[0,1.25],[1.25,3.75]$, and $[3.75,5]$; let $A=0, B=1.25, C=3.75$, and $D=5$; stepsize for each subinterval is

$$
\begin{aligned}
& \Delta x_{1}=\frac{(B-A)}{M / 2}, \\
& \Delta x_{2}=\frac{(C-B)}{M}, \\
& \Delta x_{3}=\frac{(D-C)}{M / 2} .
\end{aligned}
$$

The coordinates of the grid points are as follows.

$$
\begin{aligned}
& \text { For } j=1,2, \ldots,(M / 2)+1 \\
& \qquad x(j)=A+(i-1) \Delta x_{1} .
\end{aligned}
$$

For $j=1,2, \ldots, M$

$$
x\left(j+\frac{M}{2}+1\right)=B+(i) \Delta x_{2} .
$$

For $j=1,2, \ldots, M / 2$

$$
x\left(j+\frac{3 M}{2}+1\right)=C+(i) \Delta x_{3} .
$$

And collocation points are as follows.

$$
\begin{aligned}
& \text { For } j=1,2, \ldots, 2 M \\
& \qquad x_{c}(j)=\frac{x(j)+x(j+1)}{2} .
\end{aligned}
$$

Temperature $y_{\mathrm{Haar}}$ at higher interval, $[0,5]$, by Haar wavelet-quasilinearization technique at $J=5$ and iteration $n=4$ of the cooling equation for different values of $\varepsilon$ is shown in Figure 2. It shows that temperature decreases with increasing $\varepsilon$ and also shows that temperature reduces to zero when time $x$ is increasing. According to Table 3, we conclude that our results are in good agreement with exact solution and more accurate than variational iteration method $y_{\mathrm{VIM}}[11]$ and homotopy perturbation method $y_{\mathrm{HPM}}[11]$.

We can get more accurate results while increasing level of resolution $J$, iteration $n$, or both, according to convergence analysis [6]. 
TABLE 3: Numerical results for cooling equation for different $\varepsilon$ and $x=0.5$ : Haar wavelet-quasilinearization technique at 4 th iteration and level of resolutions $J=8$.

\begin{tabular}{lcccc}
\hline & & 4 th iteration $(J=8)$ & & \\
$\varepsilon$ & Exact & $y_{\mathrm{VIM}}[11]$ & $y_{\mathrm{HPM}}[11]$ & 0.606531 \\
0.0 & 0.606531 & 0.591638 & 0.606531 \\
0.1 & 0.606531 & 0.591617 & 0.578371 & 0.591592 \\
0.2 & 0.591591 & 0.578207 & 0.566732 & 0.578023 \\
0.3 & 0.578023 & 0.566185 & 0.556720 & 0.565620 \\
0.4 & 0.565620 & 0.555440 & 0.548335 & 0.554217 \\
0.5 & 0.554217 & 0.545868 & 0.541576 & 0.533681 \\
0.6 & 0.543681 & 0.537369 & 0.536445 & 0.524793 \\
0.7 & 0.533903 & 0.529850 & 0.532940 & 0.516275 \\
0.8 & 0.524793 & 0.523226 & 0.531062 & 0.508284 \\
0.9 & 0.516275 & 0.517412 & 0.530812 & 0.500765 \\
1.0 & 0.508284 & 0.512333 & & \\
\hline
\end{tabular}

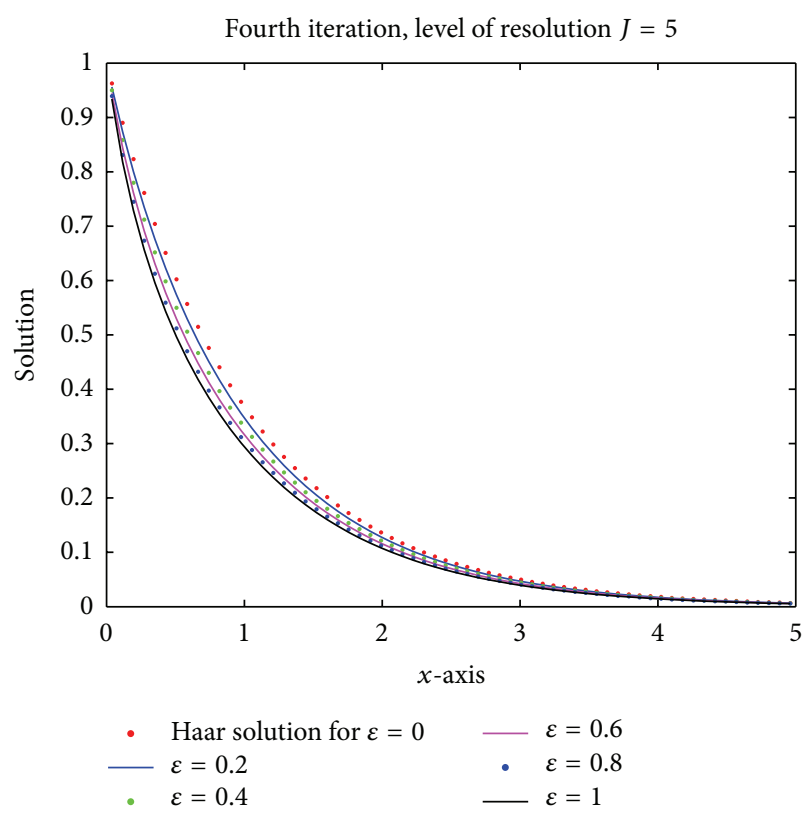

FIGURE 2: Solutions by Haar wavelet-quasilinearization technique for different $\varepsilon$ at $J=5$ and $n=4$.

\section{Conclusion}

It is shown that Haar wavelet method with quasilinearization technique gives excellent results when applied to different nonlinear heat transfer problems. The results obtained from Haar wavelet-quasilinearization technique are better from the results obtained by other methods and are in good agreement with exact solutions.

\section{Conflict of Interests}

The authors declare that there is no conflict of interests regarding the publication of this paper.

\section{References}

[1] I. Daubechies, "The wavelet transform, time-frequency localization and signal analysis," IEEE Transactions on Information Theory, vol. 36, no. 5, pp. 961-1005, 1990.

[2] R. E. Bellman and R. E. Kalaba, Quasilinearization and Nonlinear Boundary-Value Problems, Elsevier, New York, NY, USA, 1965.

[3] H. Kaur, R. C. Mittal, and V. Mishra, "Haar wavelet quasilinearization approach for solving nonlinear boundary value problems," American Journal of Computational Mathematics, vol. 1, pp. 176-182, 2011.

[4] H. Kaur, R. C. Mittal, and V. Mishra, "Haar wavelet quasilinearization approach for solving laneemden equations," International Journal of Mathematics and Computer Applications Research, vol. 2, pp. 47-60, 2012.

[5] R. Jiwari, "A Haar wavelet quasilinearization approach for numerical simulation of Burger's equation," Computer Physics Communications, vol. 183, no. 11, pp. 2413-2423, 2012.

[6] U. Saeed and M. Rehman, "Haar wavelet-quasilinearization technique for fractional nonlinear differential equations," Applied Mathematics and Computation, vol. 220, pp. 630-648, 2013.

[7] R. L. Burden and J. D. Faires, Numerical Analysis, Brooks/Cole Thomson Learning, 2001.

[8] I. Podlubny, Fractional Differential Equations, Academic Press, San Diego, Calif, USA, 1999.

[9] C. F. Chen and C. H. Hsiao, "Haar wavelet method for solving lumped and distributed-parameter systems," IEE Proceedings Control Theory \& Applications, vol. 144, no. 1, pp. 87-94, 1997.

[10] R. A. Khan, "Generalized approximation method for heat radiation equations," Applied Mathematics and Computation, vol. 212, no. 2, pp. 287-295, 2009.

[11] D. D. Ganji and A. Sadighi, "Application of homotopy-perturbation and variational iteration methods to nonlinear heat transfer and porous media equations," Journal of Computational and Applied Mathematics, vol. 207, no. 1, pp. 24-34, 2007. 

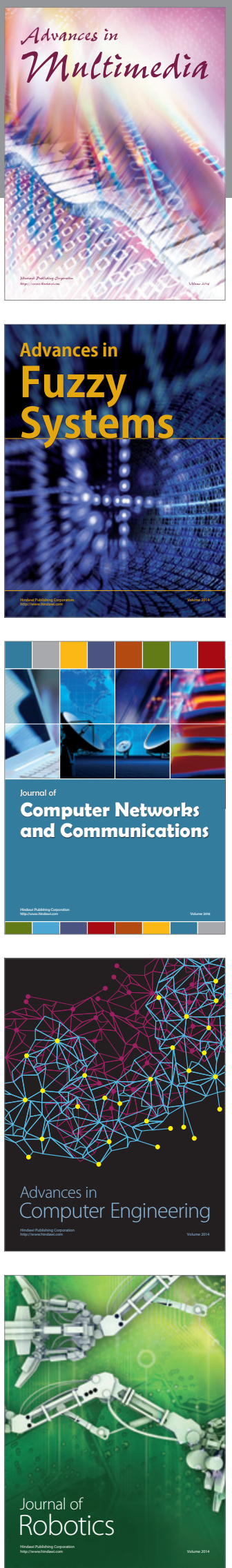

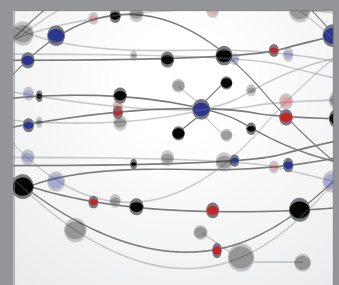

The Scientific World Journal
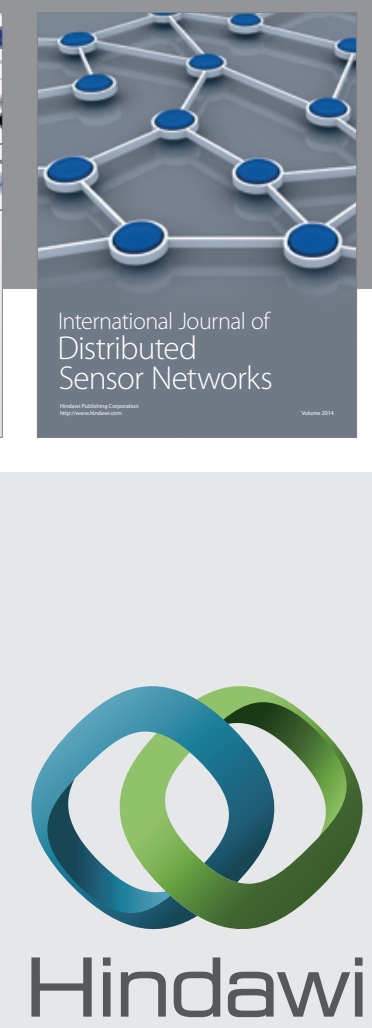

Submit your manuscripts at

http://www.hindawi.com
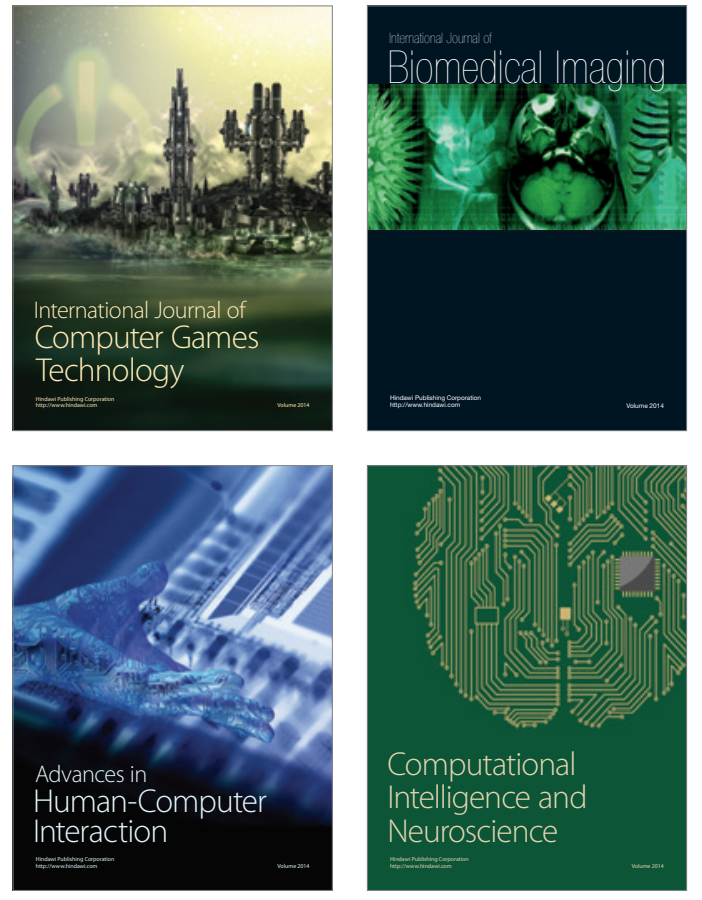
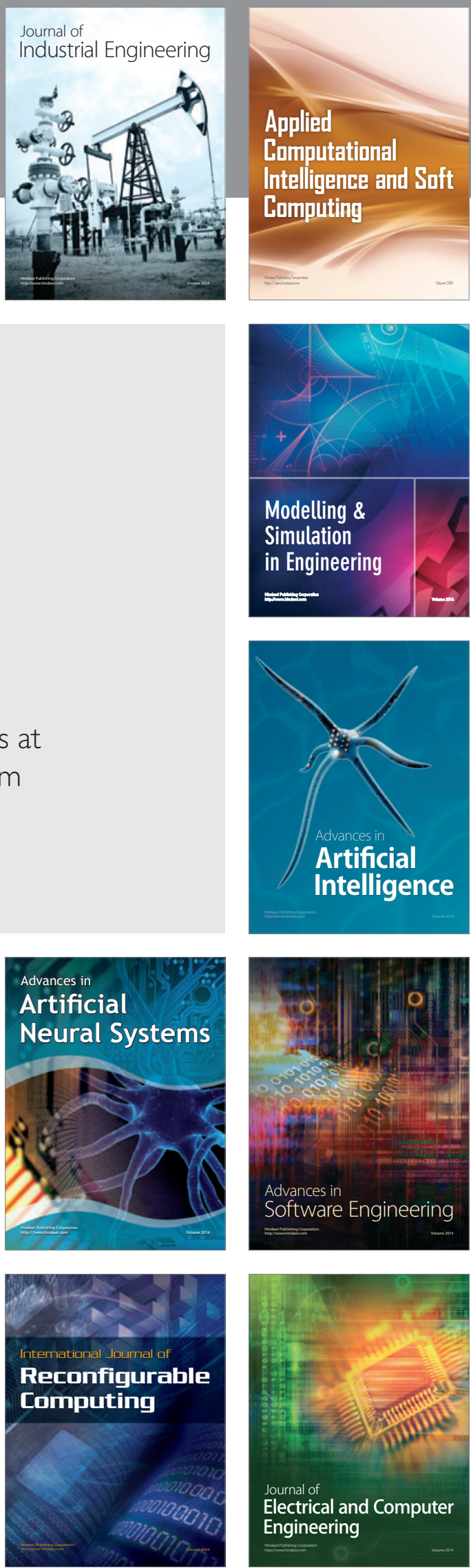